\title{
Nanoparticles-photons: effective or defective nanomedicine
}

\begin{abstract}
Different types of nanoparticles (tiny-sized particles) comprising atoms of suitable elements are being used with coating or bare surfaces for various biological and medical applications including the stent ones. The author's recent studies show that atoms of tiny-sized particles elongate or deform depending on the mode of impinging electron streams from the external source or the mode of process of synergy. Prior to those, atoms elongate under the influence of exerting forces of surface format when they are at the required level. But, atoms of tiny-sized particles report their certain impact when executing electron-dynamics. So, those elongation and deformation behaviors of atoms of tiny-sized particles may introduce the pronounced effects while their usage as nanomedicine application (or other) either in effective manner or in defective manner.
\end{abstract}

Keywords: materials science, nanoscience, nanotechnology, tiny-sized particles, atomic behavior, electron-dynamics, Nanomedicine, nanoparticles, photon energy
Volume 5 Issue 6 - 2017

\author{
Mubarak Ali \\ Department of Physics, COMSATS University Islamabad \\ Correspondence: Mubarak Ali, Department of Physics, \\ COMSATS University Islamabad, Pakistan, \\ Email mubarak74@mail.com
}

Received: April 20, 2017| Published: July 27, 2017

\section{Introduction}

Study of nanoparticles at atomic level is vital to implant their usage as a nanomedicine. As for effective drug delivery utilization, a size, shape, and density of nanoparticles along with their surface functionality are important factors and the kinetics of drug releasing can be tuned under the selection of the nanoparticles. ${ }^{1}$ Several studies are available reviewing the metallic nanoparticles' scope in relation to various medical applications..$^{2-4}$ Potential benefits of the nanoparticles are accepted in smart drug delivery and diagnostic systems along with coronary restenosis. ${ }^{5}$ Darren and Chithrani $^{6}$ presented a comprehensive review on applications of nanoparticle in medicine by offering view point on visualization, diagnosis and treatment of disease under a variety of nanoparticles. Yang ${ }^{7}$ developed a novel biliary stent. Assembling of colloidal matter into a structure having meaningful characteristics will result into treat atoms and molecules as materials for tomorrow, ${ }^{8}$ however, for that, to understand dynamics of developing of tiny-sized particles at individual basis is the key. ${ }^{9}$ The development mechanisms of variety of tiny-sized particles were discussed where the role of van der Waals interactions in binding atoms negated along with phenomenon of surface plasmons. ${ }^{10}$ In suitable atoms of solid where electron-dynamics under conservative forces are dealt, their binding is by means of engaged conserved energy. ${ }^{11}$ The study of tiny-shaped particles developing mono-layer dealing localized gravity at solution surface is discussed; ${ }^{12}$ when a tiny particle developed in joint triangles, exerting force along slightly perturbed axis divided them at the point of connection. Developing tiny-sized particles and their large-sized particles under varying the precursor concentration is also studied. ${ }^{13} \mathrm{~A}$ tailored nano shape energy under tuned bipolar pulse resulted into convert monolayer assembly of transition state gold atoms into own shape and the triangularshaped tiny particles developed directly under unipolar mode of pulse polarity. ${ }^{14}$ Causes have been explored to tap such tiny-shaped particles in silver and binary composition as well while employing the same setup as for the case of gold precursor. ${ }^{15}$

Under the evidences of experimental results, the conventional binding mechanism of atoms and light-matter interaction is negated where the duration to process a solution for developing tiny particles and their extended particles is discussed. ${ }^{16}$ Solid atoms of electron transition, on impinging electron streams from the external source or under the process of synergy also, elongate or deform but do not ionize. ${ }^{17}$ Silicon atoms of neutral behavior when transform heat energy into photons of wavelength of current, they are related to photonic current when their certain density propagate through inter-state electron gaps of suitable wire. ${ }^{18}$ Origin of atoms to be in gas state and solid state is being explored establishing relationship in their force-energy behavior. ${ }^{19}$ Elongation of atoms in tiny-sized particles is taken place when the stretching of clamped energy knots to electrons is orientationally based, whereas, deformation of atoms is under the stretching of clamped energy knots to electrons in nonorientationally manner. ${ }^{17}$ Therefore, modifications of certain behavior atoms under their inherently built-in scheme of electrons are the usual case. Tiny-sized particles mainly address to those atoms which are remained grounded (buried) at below average-leveled ground surface and their state is related to solid. However, their tiny-sized particles are developed at average-leveled ground surface when dealing certain transition state, so, their atoms deal exerting forces of surface format at electron levels. ${ }^{12}$

Modifications of atoms are under their elongation and deformation processes. Such modifications, in either way, don't retain the original behavior of atoms in their tiny-sized particles. When atoms of certain elements are in their neutral state, they execute electron-dynamics under the conservative mode of force where transforming the heat energy into photon energy. ${ }^{11,18}$ Therefore, elongated or deformed atoms of tiny-sized particles don't remain in functioning in terms of transforming the heat energy into photon energy upto the desired (expected) level. Again, exerting forces at electron levels for atoms of tiny-sized particles may influence a bit in fluctuating manner resulting into disturb the regularity of electron-dynamics, thus, their use for the certain nanomedicine treatment may be either effective, less effective or defective. As an example, a precise structure of tiny-sized particle maintains its stent application where that prevents the arterial closings, thus, keeps it open under the adequate expansion through the application of generated featured photon energy. Here, expansion is being maintained by the featured photon energy. The featured photon energy generated by the gold atom is different than that of silicon atom because of exerting different nature of electron-dynamics. Atoms of those tiny-sized particles might generate the targeted energy having different features because some of the atoms might deform or elongate 
not enabling the execution of electron-dynamics. Then, that featured photon energy can either be under-estimated or be over-estimated from the exact estimation. So, in the case of functioning of tiny-sized particles (or their atoms) in defective manner, instead of curing the disease, they themselves become the causes of disease resulting into worsen the disease, thus, lowering the survival rate of patient under the treatment. Atoms of certain elements deform or elongate as per available room in terms of unfilled states and at the point where no longer stretching of energy knots clamped electrons is remained, they start to erode. ${ }^{17}$

Such modifications of atoms become effective in making their tiny-sized particles defective or they work in less-effective manner because, the electron(s) at the outer ring of atom are not in their natural confinement and they are not delivering the necessary quantized energy (photons) in the manner required at (to) the point of cure. Such behaviors are quite diligent in atoms of tiny-sized particles belonging to elements known in their magnetic behavior or metallic behavior. In the case, where atoms of tiny-sized particles are functioning well in terms of their electron-dynamics, it is fine, if required level of energy has been pre-determined at the point of cure. Three different tiny-sized particles determining effective energy of photons are shown in Figure $1(\mathrm{a}-\mathrm{c})$. Three different destructive or less-effective tinysized particles determining the energy are shown in Figure 2 (a-c). In Figure 1 (a), a mono-layer three-dimensional structure is bound to determine controlled behavior of force-energy (photon) per unit area. In Figure 1 (b), atoms of one-dimensional arrays converted into structures of smooth elements determining enhanced propagation of forcing energy (photons) per unit surface area, thus, its functioning is different from the one shown in Figure 1 (a). In Figure 1 (c), an ordered structure of tiny-sized particle other than shown in Figure 1 (a) and 1 (b) determines different photon energy, which is in unit volume. In Figure 2 (a-c), all three different tiny-sized particles don't show uniform distribution of energy either per unit area or per unit volume, thus, working as defective or less-effective nanomedicines. These tiny-sized particles are called nanoparticles because quite a large sized particle $(>15 \mathrm{~nm})$ is also termed as 'nanoparticle'. For nanomedicine applications, the requirement of the size is in the range of $5 \mathrm{~nm}$ to $15 \mathrm{~nm}$, in most cases. A forcing energy is related to the photon energy (Figure 1)(Figure 2). ${ }^{18}$

When atoms of tiny-sized particles don't retain their original ground point, which is, in fact, at below ground surface, they deal transition in state where exertions of ground forces elongate them. Further details of elongation behavior in the case of tiny-shaped particles ${ }^{12}$ and in the case of standalone atom are given elsewhere. ${ }^{17}$ When tinysized particles (their atoms) deal impinging electron streams or the process of synergy under different interactions, their deformation and elongation behaviors also deal the forcing (forced) energy but carrying (carried) by the electrons (or electron streams). ${ }^{10-18}$ The atoms of tiny-sized particles absorb heat energy in the form of tits and bits where transforming them into the force-energy (photons), thus, those energies are supplied to the infected region needing treatment or doze and, also, where it is not possible in a natural sort of way. Under defective structure of tiny-sized particles or incompetence utilization of those effectively delivered energies at (to) the point of cure can result into unavoidable medical issues to the patient which may also not be cured under certain circumstances. Thus, those tinysized particles or their certain number of atoms or even their single atom in a modified behavior may result into give a different result from the ones anticipated by the Surgeon or Medical specialist. Again, a bit fluctuating exerting forces for electrons disturb the regularity of electron-dynamics.

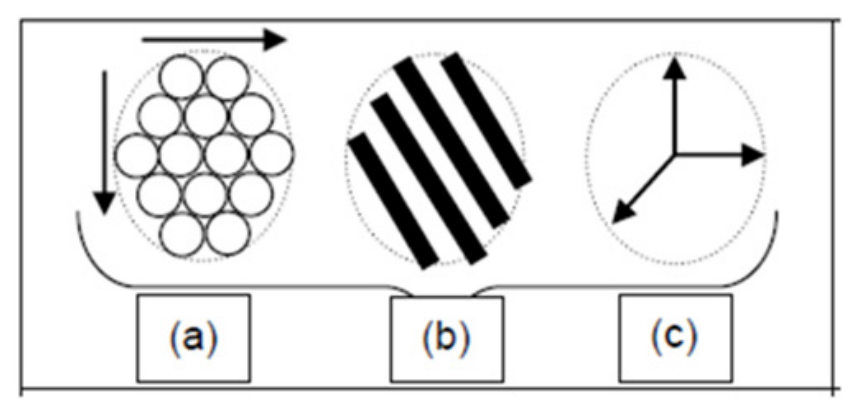

Figure I Effective nanoparticles (tiny-sized particles).

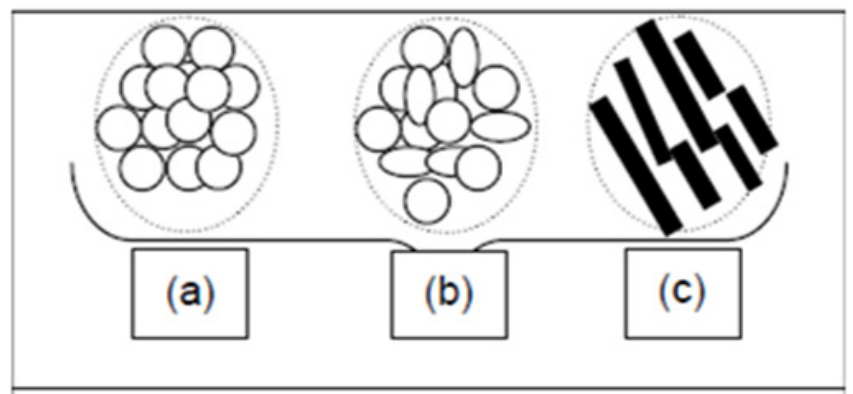

Figure 2 Defective nanoparticles (tiny-sized particles).

As a future direction, tiny-sized particles (nanoparticles) are replacing with the others in various fields of biology and medicine, working as nano-devices, where their atoms involve magnetic behavior, thus, releasing the drug load to the tumor or destroying unwanted tissue through heat as pointed out elsewhere. ${ }^{20}$ However, atomic behavior is quite sensitive where tiny-sized particles are known by their nanomedicine application as their electrons are highly sensitive to naturally exerting forces,${ }^{19}$ thus, may cause effects unexpected and not thought while their use as a nanomedicine. The effects of exerting forces at electron levels in atoms of tiny-sized particles may vary a bit region to region and may be inevitable during treatment of the patient even in a sterile room made for such purposes and under the normally taken precautions. The energy-dependent behavior of atoms is recognized where exerting relevant poles forces remain in their neutral behaviors, they are with more clarity in the case of a carbon element, which originates several different physical behaviors of a gas state atom under transferring electrons of filled states to certain unfilled states. ${ }^{21,22}$ But, how to control the behavior (s) of exerting force (s) which are influencing atoms of tiny-sized particles at electron levels is a main challenge. However, we can see and observe ice layers developing at the surface of water by itself in certain cold regions where water below the ice layer is in normal condition and aquatic life remains alive because of the not developing of further thick ice-layer as it doesn't grow further on non-exertion of gravitational force. A gravitational force doesn't exert at electron levels further because of developed ice-layer in certain thickness. Thus, the exerting gravitational force can be planned on similar lines to instate the required behavior of atoms of tiny-sized particles when using them for certain nanomedicine application.

\section{Conclusion}

Originating the certain scope of nanoscale materials for nanomedicine applications as well as for others should be based on the behavior of their comprised atoms along with the nature of executing their electron-dynamics. Such efforts to synthesize tinysized particles and their applicability will lead into their controlled usage as per their requirement, thus, resulting into effective use and 
with full confidence of medical doctor, who is, in fact, neither a materials scientist nor the one working on physical/chemical sciences, but the implications are being faced in the case of unsuccessful result of operation or treatment. In the view of above-said, scientists and researchers should research materials for nanomedicine applications and others in more planned ways by considering the original behavior and nature of their atoms, procedure of their structure evolution and, then, the scheme of structure evolution and, finally, the influence of environment (surrounding) during their applicability and by cuttingdown the financial interest as it is a matter of human health and life.

\section{Acknowledgments}

None.

\section{Conflicts of Interest}

None.

\section{Funding}

None.

\section{References}

1. Luderer FK, Sternberg HW, Rohm M, et al. Suitability of nanoparticles for stent application, IFMBE Proceedings. 2008;22:2339-2342.

2. Cai W, Gao T, Hong H, et al. Applications of gold nanoparticles in cancer nanotechnology. Nanotechnol Sci Appl. 2008;1:17-32.

3. Boisselier E, Astruc D. Gold nanoparticles in nanomedicine: preparations, imaging, diagnostics, therapies and toxicity. Chem Soc Rev. 2009;38:1759-1782.

4. Arvizo R, Bhattacharya R, Mukherjee P. Gold nanoparticles: Opportunities and Challenges in Nanomedicine. Expert Opin Drug Deliv. 2010;7:753-763.

5. Yin RX, Yang DZ, Wu JZ. Nanoparticle Drug- and Gene-eluting Stents for the Prevention and Treatment of Coronary Restenosis. Theranostics. 2014;4(2):175-200.

6. Darren Y, Chithrani BD. Applications of Nanoparticles in Nanomedicine J Biomed Nanotechnol. 2015;10:2371-2392.

7. Yang F, Ren Z, Chai Q, et al. A novel biliary stent coated with silver nanoparticles prolongs the unobstructed period and survival via antibacterial activity. Sci Rep. 2016;6:21714.
8. Glotzer SC, Solomon MJ. Anisotropy of building blocks and their assembly into complex structures. Nature Mater. 2007;6(8):557-562.

9. Link S, El-Sayed MA. Shape and size dependence of radiative, nonradiative and photothermal properties of gold nanocrystals. Int Rev Phys Chem. 2000;19(3):409- 453.

10. Ali M, Lin, IN. The effect of the Electronic Structure, Phase Transition and Localized Dynamics of Atoms in the formation of Tiny Particles of Gold. Materials Science. 2016;1:1604.07144.

11. Ali M. Structure evolution in atoms of solid state dealing electron transitions. Condensed Matter. 2016;1:1611.01255.

12. Ali M. The study of tiny shaped particle dealing localized gravity at solution surface. Materials Science. 2016;1:1609.08047.

13. Ali M, Lin IN. Development of gold particles at varying precursor concentration. Materials Science. 2016;1:1604.07508.

14. Ali M, Lin IN. Controlling morphology-structure of particles at different pulse rate, polarity and effect of photons on structure Materials Science. 2016;1:1605.04408

15. Ali M, Lin IN. Tapping opportunity of tiny shaped particles and role of precursor in developing shaped particles. Materials Science. 2016;1:1605.02296

16. Ali M, Lin IN. Formation of tiny particles and their extended shapes - origin of physics and chemistry of materials. Condensed Matter 2016;1:1605.09123.

17. Ali M. Atoms of electronic transition deform or elongate but do not ionize while inert gas atoms split. Materials Science. 2016;1:1611.05392.

18. Ali M. Revealing the Phenomena of Heat and Photon Energy on Dealing Matter to Atomic level, Preprints, Switzerland. 2017.

19. Ali M. Why some atoms are in gaseous state and some in solid state but carbon works on either side. 2017.

20. Salata O. Applications of nanoparticles in biology and medicine. J Nano biotechnol. 2004;2(1):3

21. Ali M, Lin I Nan. Phase transitions and critical phenomena of tiny grain thin films synthesized in microwave plasma chemical vapor deposition and origin of $\mathrm{v} 1$ peak. 2016

22. Ali M, Ürgen M. Switching dynamics of morphology-structure in chemically deposited carbon films -A new insight. Carbon. 2017;5:1605.00943. 